\title{
La incapacidad temporal: hacia un modelo de gestión
}

\author{
C. Navarro Arribas, S. Chicano Díaz ${ }^{1}$ \\ $J$ efe de la Dependencia Provincial de Trabajo y Asuntos Sociales de Cuenca. \\ 'Enfermera Subinspectora. Dirección Provincial INSALUD de Albacete
}

\section{RESUMEN}

El actual modelo de gestión de la incapacidad temporal cuenta con tres organizaciones con competencias sobre la misma. Se ha creado un modelo con ambiente turbulento que hace a dichas organizacio nes menos aptas para la consecución de sus objeti vos.

Apartándonos de modelos cuantitativos y econo mistas, proponemos un modelo de gestión basado en criterios científicos que nace y termina en el propio Sistema Sanitario como lo hace efectivamente la propia incapacidad temporal.

Considerando la incapacidad temporal como una prescripción médica, su evaluación y control debe ría recaer en el propio Sistema Sanitario, el mejor conocedor de su origen, evolución y de todos sus factores asociados.

Palabras clave: Incapacidad temporal. Gestión. Evaluación.
Temporary incapacity: towards a management mo del

\section{ABSTRACT}

Currently, there are three different organizations with responsibilities about the management of tem porary incapacity. This management system has lead to a turbulent atmosphere that creates serious diffi culties for these organizations to reach their targets. So we propose a management model that differs from quantitative and economic models. It is based on scientific criteria and has been developed within the Nacional Health System.

Considering temporary incapacity as a medical prescription, its control and evaluation should rely on the Health System which is the organization that best know the origin and evolution of temporary in capacity as well as all other factors related with it.

Key words: Temporary incapacity. Management. Evaluation.

\section{INTRODUCCIÓN}

La incapacidad temporal (IT) se define por primera vez en nuestro Ordenamiento Jurídico con la Ley 42/94 de 30 de diciembre de Acompañamiento a los Presupuestos. Sustituye al anterior concepto de Incapacidad Laboral Transitoria y queda definida como "aquella situación en la que se encuentran los trabajadores impedidos temporalmente para trabajar debido a enfermedad común o profesional y accidente, sea o no de trabajo, mientras reciban asistencia sanitaria de la Seguridad Social, así como los periodos de observación por enfermedad profesional en los que se prescriba la baja en el trabajo durante los mis- 
mos". Su duración máxima es de 12 meses prorrogables por otros seis cuando se presuma que durante ellos pueda el trabajador ser dado de alta médica por curación ${ }^{1-3}$.

Sobre la consideración de una prestación del subsistema de la Seguridad Social tendente a sustituir las rentas dejadas de percibir por el trabajador, se han implantado tradicionalmente las medidas de inspección y control dirigidas a contener el incremento económico que progresivamente ha experimentado.

Entre estas medidas destacan, posibilitadas por los cambios normativos que la Ley 42/1994 promulgó, la participación de los Servicios Médicos del Instituto Nacional de la Seguridad Social y de las Mutuas de Accidente de Trabajo. Se ha producido en este contexto, un aumento de los reconocimientos médicos a pacientes en IT y una regularización de los ficheros administrativos que han conseguido en una primera fase, la corrección de los indicadores de gestión de la $\mathrm{IT}^{4}$.

Sin embargo, esta estrategia de control no ha sido capaz de mantener su eficacia produciéndose un importante repunte de los indicadores (Figs. 1 y 2).

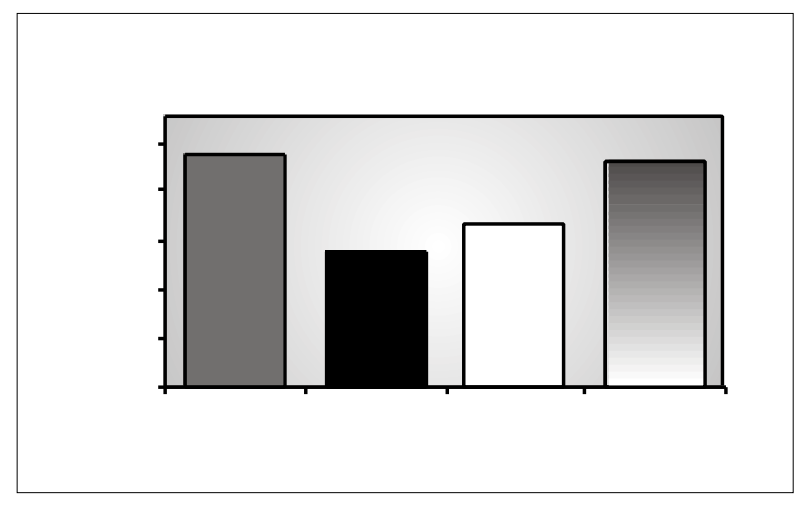

Figura 1

Representación de la evolución de los días de baja por enfermedad durante los años 1997 a 2000 en el Siste-

ma Nacional de Salud.

Nos enfrentamos a uno de los capítulos mayores de la Atención Primaria con importantes repercusiones sobre el Sistema Sanitario debido al importantísimo coste que, de forma además creciente, repercute en el subsistema de la Seguridad Social y paralelamente a la financiación sanitaria ${ }^{5}$. Dicho coste ha sido calculado en aproximadamente el $50 \%$ del gasto anual de un Equipo de Atención Primaria de unos 18.000 habitantes y el absentismo por incapacidad temporal tiene un coste directo e indirecto que puede cuantificarse en más de 12.000 millones de e anuales sin contar el gasto de pensiones por invalide $\mathrm{z}^{6,7}$. El coste por IT que ha de soportar la entidad gestora se aproximaría a los 6 millones de e anuales para un Ârea de Salud de aproximadamente doscientos mil usuarios que suponen en la provincia de Cuenca

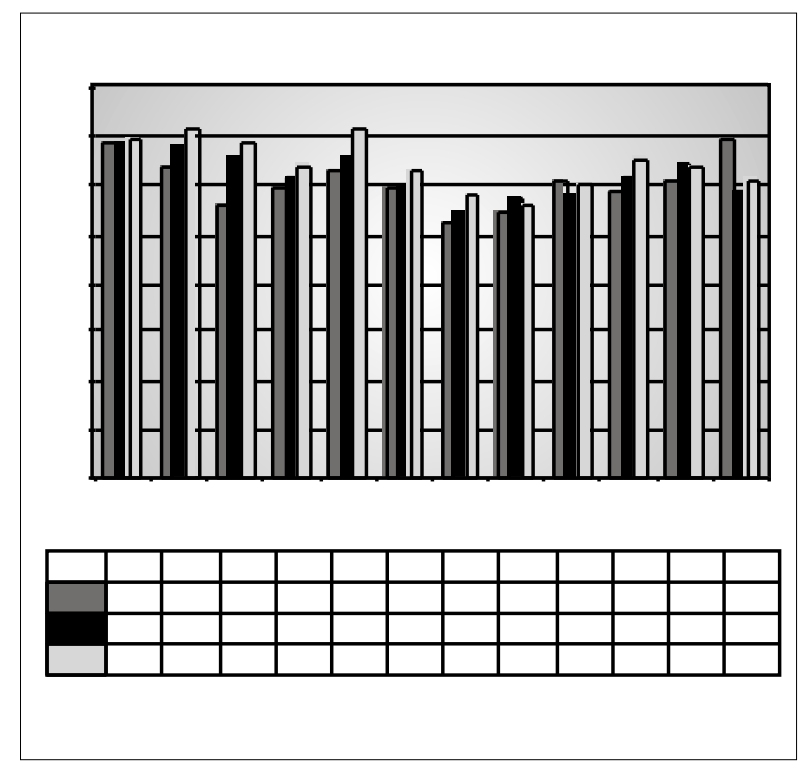

Figura 2

65.000 trabajadores activos con derecho a prestación económica por IT; es decir, más de 90 e /año por persona con derecho a IT, superior si cabe al coste de la prestación farmacéutica. Por ello, sin prescindir de ninguna medida de control sería preciso habilitar nuevas formas de actuación que prevengan la aparición de las bajas ${ }^{8}$, especialmente si consideramos que dos tercios de la IT corresponderían a simuladores y a demoras al acceso a Atención Especializada9.

El sujeto que se encuentra en situación de incapacidad temporal es, especialmente en los casos derivados de accidente, un adulto sano que de forma más o menos brusca pierde su capacidad funcional, incluso su propia autonomía y en muchas ocasiones merma su capacidad de ganancia. El trabajador entra con demasiada frecuencia en conflicto con el empresario que a su vez a de soportar costes añadidos en la gestión de personal. Este último hecho es especialmente válido para las pequeñas y medianas empresas que en muchas regiones son la base de la actividad económica.

Pero además son muchos los factores que influyen en la obtención y/o prolongación indebida de la IT: insatisfacción laboral, problemática sociofamiliar, prolongación de desempleos, despidos pactados, cuidado de hijos, regímenes sin prestación por desempleo, dificultad de acceso a Nivel Especializado, escasa respuesta de este nivel hacia la Primaria, masificación de consultas de Medicina General, escasa respuesta de la Inspección de los Servicios Sanitarios, falta de concienciación de la repercusión real de la prescripción de una baja laboral, derivación de bajas procedentes de contingencias profesionales $^{10}$.

Todos estos factores tienen que ser amortiguados por el facultativo de Atención Primaria en un contexto organizacional que podemos definir como turbulento $^{11}$. Efectivamente, a la presión que ejerce el 
paciente, tendríamos que añadir la de otras Organizaciones con competencia sobre la gestión de la IT (Servicio de Salud, INSS, mutuas). Al facultativo de Primaria se le hace responsable de las bajas laborales pero no obtiene ningún beneficio directo o indirecto de su gestión lo que origina una clara desmotivación en aras de la actividad puramente asistencial. Además el facultativo de este nivel no ha recibido formación en materia de valoración de la incapacidad. Un repaso a los programas de las distintas especialidades y tratados de medicina ponen de manifiesto que la IT no es sistemáticamente contemplada como parte del tratamiento de las principales patologías que generan y perpetúan la IT. Otro tanto cabe decir de la duración aproximada de dichos procesos (Figs. 3 y 4). El médico se ha visto obligado a actuar empíricamente en función de su experiencia, intuición y ética, y en el momento de extender una baja o forzar un alta se ha preguntado si la decisión tomada era la correcta técnicamente o era la mejor que podía tomar en una determinada situación ${ }^{12}$. Estas circunstancias no favorecen un posicionamiento adecuado ni firme ante el trabajador que demanda una baja laboral, ni frente a las organizaciones que con mucha frecuencia le hacen injustamente responsable último y único de la IT.

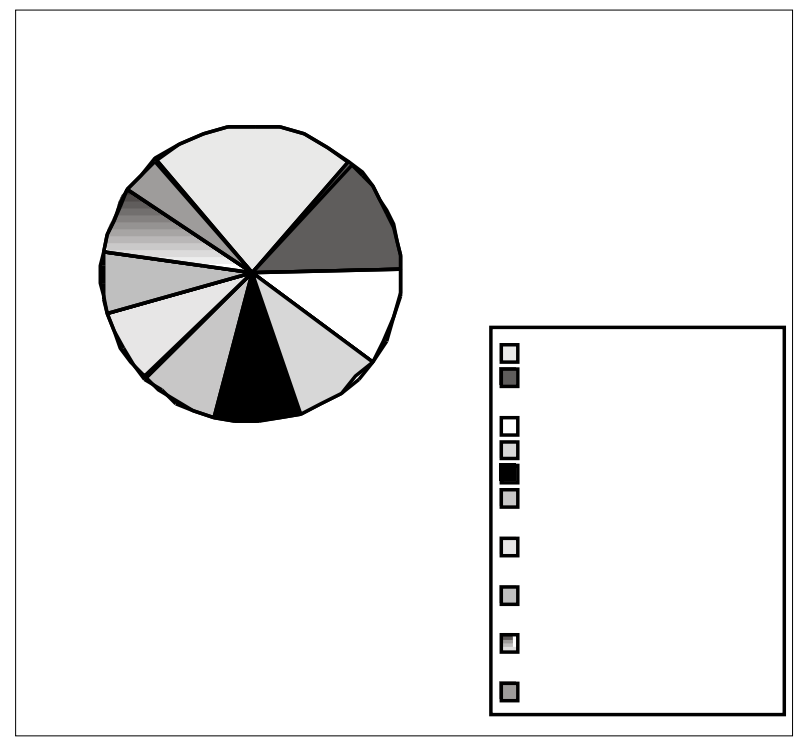

Figura 3

En este momento queremos significar que la importante aportación que para el facultativo de Primaria puede suponer el Manual de Gestión de la Incapacidad editado por el Instituto Nacional de la Salud con la colaboración y participación de las Sociedades Científicas y de los distintos profesionales implicados, lo que garantiza el consenso y el enfoque multidisciplinar de su contenido. Responde a la pregunta no siempre considerada en los tratados de medicina sobre cuándo procede y cuánto debe

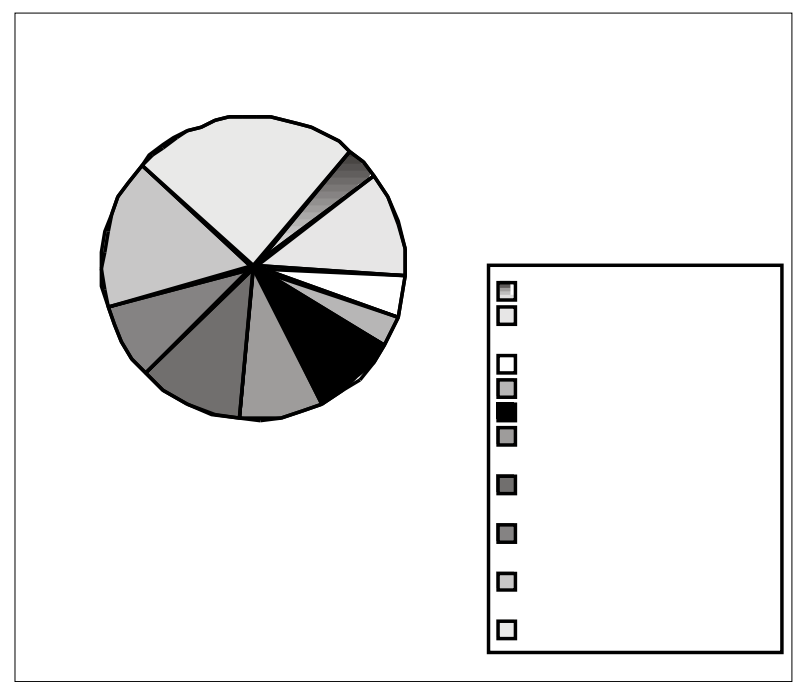

Figura 4

durar una prescripción de reposo ${ }^{13}$. Al conocimiento científico que aporta añadimos su consideración como instrumento de seguridad jurídica ya que el manual nace del consenso de la comunidad científica y podría adquirir la consideración de protocolo especialmente si su contenido es consensuado por los equipos de Atención Primaria ${ }^{14}$. Recordemos la alta judicialización de la IT cuyas reclamaciones podrían alcanzar mas del $30 \%$ del total de las reclamaciones presentadas en nuestro medio (Fig. 5).

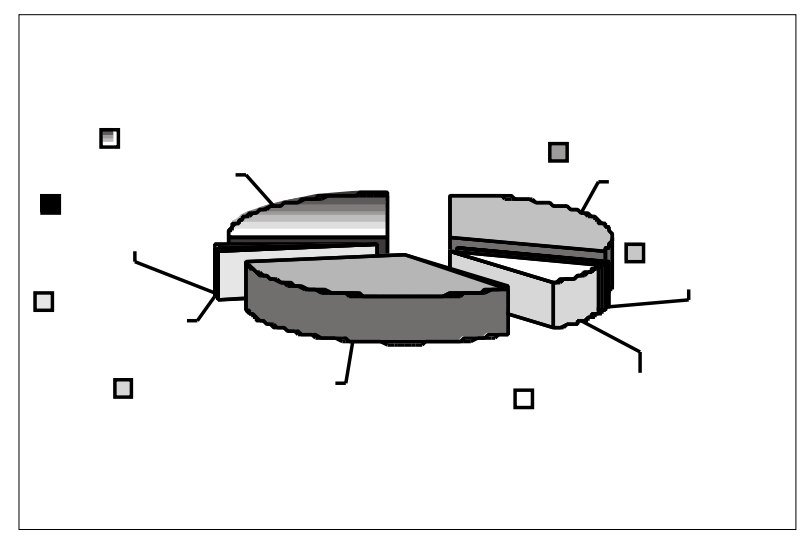

Figura 5

\section{UN NUEVO CONCEPTO}

El Sistema Nacional de Salud debe considerar la IT, no como una prestación económica de la Seguridad Social, sino como la consecuencia de un acto médico y por tanto como una prescripción médica. Es decir, consideramos la IT como parte del tratamiento que recibe el trabajador de su médico de Atención Primaria. Definimos la misma, como, la situación de 
aquel paciente que, como consecuencia de su enfermedad o accidente, presenta limitaciones funcionales, presumiblemente reversibles, que le impiden ejecutar las tareas propias de su profesión y/o el desempeño de tales tareas repercutirían negativamente en la evolución favorable de su patología.

Que la duración de IT tenga por norma un máximo de 12 meses choca frontalmente con criterios científicos. Salvo que imperen exclusivamente razones económicas estrictas, la duración debería ser aumentada más allá de los 6 meses que propone la legislación de la Ley General de la Seguridad Social, con objeto de promover un cambio de situación (alta, informe propuesta de invalidez) basado en criterios clínicos-funcionales.

Desde el concepto que proponemos, la indicación de IT tendría la misma consideración que la prescipción de cualquier procedimiento diagnóstico, terapéutico, rehabilitador o preventivo. Por ello las medidas de control de las situaciones que ocasionan las bajas laborales implicarían al Sistema de Salud y a sus recursos humanos y materiales consiguiendo que la incapacidad:

-Fuera una parte no diferenciada del resto del tratamiento que recibe el paciente eludiendo así las críticas que las actuales medidas de control han recibido desde distintos sectores sociales.

-Que su gestión se basara en criterios de gestión sanitaria.

\section{EXPERIENCIASJUSTIFICATIVAS}

Consideramos que la duración de los procesos de IT por hernia inguinal no se ven modificados como consecuencia de los reconocimientos médicos de control. En la fase previa al tratamiento quirúrgico de la hernia, el paciente presenta una patología claramente objetivable que ha justificado la prescripción de IT por su médico de Atención Primaria. El paciente además se encuentra incluido en lista de espera quirúrgica como único procedimiento de resolución de su patología. Una vez ha sido intervenido, en nuestro medio es el cirujano quien indica al médico de Atención Primaria el momento del alta laboral en función de la evolución y profesión del paciente. El alta laboral se indica a los 30-60 días de la intervención.

Los procesos por hernias inguinales han sido incluidos en el programa de lista de espera quirúrgica en los años 1998 y 1999 en el Área de Salud de Cuenca: se ha conseguido una notable reducción de la demora quirúrgica que ha pasado de 118 días en 1997 a 80 días en el año 1998 y a 59 días en 1999. Han sido intervenidos un total de 22 pacientes en el marco de este programa ${ }^{15}$.

Paralelamente la duración media de la IT por hernia inguinal ha experimentado una reducción de 20 días respecto al año 1997. Se ha pasado de 80,4 a 60,29 días.
En ausencia de cualquier otra intervención, sobre la duración media de la incapacidad por hernia inguinal, que hemos podido comprobar que no ha existido, parece razonable afirmar que ha sido una mejora de la eficacia del Sistema Sanitario la responsable de esta reducción, en este caso por programas de lista de espera quirúrgica.

Experiencias en este sentido han sido revisadas en patologías reumatológicas donde una intervención precoz de facultativos especialistas de Área en apoyo del médico de Atención Primaria han conseguido reducir sensiblemente la duración de la incapacidad por estas patologías ${ }^{16}$.

En otro orden de cosas, las revisiones conjuntas de la IT llevadas a cabo por la Inspección de Servicios Sanitarios con los médicos de Atención Primaria han puesto de manifiesto las siguientes circunstancias en el Área de Salud de Cuenca:

- Los diez facultativos con mejores indicadores de IT, gestionan estos procesos con la historia clínica y cuatro de ellos han cursado programas de gestión de incapacidad, por el contrario.

-Los diez facultativos con peores indicadores no utilizan la historia clínica y ninguno de ellos ha realizado cursos de IT.

Considerada la historia clínica como un instrumento de calidad de la asistencia prestada ${ }^{17}$, encontramos otro claro paralelismo entre la mejora del Sistema de Salud y la evolución de la incapacidad que nos permite afirmar que la mejora de la calidad de los Servicios Sanitarios Públicos es una estrategia clave en el control de la IT.

\section{MODELO DE GESTIÓN}

Consideramos que la gestión de IT ha de nacer del propio Sistema Nacional de Salud y se basaría en las siguientes premisas:

-Mejoras en la reducción de las listas de espera quirúrgica y de acceso a consulta de Atención Especializada. Ambos son objetivos institucionales del Sistema Nacional de Salud que beneficiaría directamente la tendencia de la IT.

-Formación, información y motivación de los facultativos, tanto de Atención Primaria como de Especializada.

-Pactos en los contratos de gestión de Atención Primaria.

-Evaluación y retroalimentación del modelo.

Nuestro modelo de gestión tendría como punto de partida el Manual de Gestión de la Incapacidad Tempo $\mathrm{ral}$, al que ya nos hemos referido y cuya tercera y última edición de 2001 amplía considerablemente el número de procesos cuya duración queda estandarizada. La selección de dichos procesos se ha basado en registros de incidencia cubriendo la mayor parte de las patologías que ocasionan situaciones de IT.

El contrato de gestión, constituye el segundo instrumento de gestión. En él se establecerían los obje- 
tivos comunes para todos los Equipos del Área. Este objetivo viene referido a dos indicadores de fácil obtención e interpretación: la prevalencia de las bajas y la duración media de las bajas ${ }^{18}$. La prevalencia relaciona por cociente los asegurados en baja a fin de mes con los asegurados activos; la duración media de la baja se obtiene dividiendo los días en baja de las altas por las altas tramitadas.

Una vez comprobado que la prevalencia y la duración media han sido alcanzadas en el conjunto del Área de Salud se procedería a evaluar los distintos equipos de Atención Primaria. Esta evaluación la llevaría a cabo la inspección médica y se centraría sobre los distintos facultativos y los procesos de incapacidad que prescriben y mantienen.

Se evaluarían por cada facultativo y año tres patologías que se seleccionarían en función de su elevada prevalencia o duración media en el Área. Por cada proceso de IT se evaluaría:

1. Si el proceso ha tenido una duración acorde a los criterios establecidos en el Manual de Gestión de la Incapacidad Temporal.

2. Si el proceso ha sido registrado en la historia clínica.

3. Si han sido emitidos partes de alta por incomparecencia a aquellos pacientes que no acuden a consulta sin causa justificada por dos partes de confirmación consecutivos.

4. Número de altas por inspección en el periodo evaluado.

El criterio 1 coincidiría con un proceso de IT justificado. El criterio 2 expresa, como hemos comentado, un elemento de calidad de la atención prestada. El criterio 3, valora el seguimiento del proceso de IT por parte del facultativo y el criterio 4 refiere la máxima expresión de una baja laboral no justificada por razones clínico-sanitarias.

Estos criterios serían valorados según la puntuación que proponemos en la tabla I, obteniéndose para cada facultativo una determinada puntuación. El encontrarse por debajo de la media de las puntuaciones de todos los facultativos del Área sería considerado como un elemento de calidad en la gestión de la de la IT y considerado junto con la valoración de la prevalencia y de la duración media como elementos para obtener las repercusiones pactadas en los contratos de gestión.

Entendemos que el modelo propuesto aporta, frente a otras medidas de control de las bajas laborales, los siguientes elementos propios y diferenciadores:

- Considera la IT como una prescipción del facultativo de Atención Primaria utilizando para lograr su adecuación instrumentos de gestión propios del Sistema Sanitario, y en especial los contratos de gestión.

- Involucra a todos los recursos humanos del Sistema: facultativos, gerentes e inspección médica.

-Los objetivos y las medidas correctoras nacen del propio Sistema Nacional de Salud, el mejor conocedor de la IT y del conjunto de todos los factores que la afectan.
Tabla I

BAREMO DE EVALUACIÓN DE INCAPACIDAD TEMPORAL

Falta de historia clínica laboral

o registros concretos del proceso de IT 1 punto

Falta de emisión del parte de alta

por incomparecencia

2 puntos

Proceso que supera la duración estándar

3 puntos

Alta por inspección médica

4 puntos

-Es factible y sencillo.

- Se aparta deliberadamente de criterios economistas, sin dejar de considerar el coste económico que supone la IT, que se alcanzaría a través de un adecuado proceso.

- Garantiza los derechos del usuario y del facultativo a través de una participación activa y directa de la inspección de Servicios Sanitarios.

- Se trata de un proceso continuo que no necesita de medidas de apoyo.

-El modelo consideraría cuatro de los factores condicionantes de la hiperprescripción de bajas: lista de espera, falta de coordinación entre niveles, falta de control por inspección médica y complacencia del propio facultativo ${ }^{19}$.

\section{CONCLUSIÓN}

Hemos propuesto un modelo de gestión de la Incapacidad Temporal que homologa la baja laboral a una prescripción médica, por ello utiliza medidas de control propias del Sistema Sanitario para la consecución de su objetivo. A estas medidas se añadiría una mejora constante del acceso a la Atención Especializada.

El modelo considera como imprescindible un programa de formación de los facultativos en materia de valoración de la Incapacidad Temporal para lograr su participación activa.

Consideramos que el modelo tendría mejor aceptación que los modelos tradicionales, pues rompería con la incertidumbre que se ha creado entre facultativos y pacientes, y lograría una mayor supervivencia a medio-largo plazo.

CORRESPONDENCIA:

Sandra Chicano Díaz

Dirección Provincial INSALUD de Cuenca

C/ Colón, 12

16071 Cuenca 


\section{Bibliografía}

1. Real Decreto Legislativo $1 / 1994$, de 20 de junio, por el que se aprueba el Texto Refundido de la Ley General de la Seguridad Social.

2. Ley 42/1999, de 30 de diciembre, de Medidas Fiscales, Administrativas y de Orden Social. BOE de 31 de diciembre de 1994.

3. Guía Laboral y de Asuntos Sociales 2001. Subdirección General de Información Administrativa de la Secretaria Técnica del Ministerio de Trabajo y Asuntos Sociales. Madrid, 2001.

4. Gálves C, Rodríguez J, García RA, Peregrina M, Torres I, Medina A, et al. Valoración del control médico de los procesos de Incapacidad Temporal en Granada, realizado por los Facultativos del INSS. Comunicación de las VIII Jornadas Nacionales de Inspección de Servicios Sanitarios; 1999 diciembre 1-3; Toledo.

5. Sánchez Bayle M. La financiación de la sanidad. Jano 1998; 1242: 22.

6. Ruiz Téllez A. El problema de la Incapacidad Temporal y la Atención Primaria. La Gran oportunidad. La gran amenaza. Propuesta de un cambio. En: Grupo de trabajo semFYC. Incapacidad Temporal: encrucijada ética, clínica y de gestión. Documento SemFYC 12, 1999. p. 16-57.

7. Quijaro Terán F. Incapacidad Temporal/Permanente. Situación actual en España. Cambios y consecuencias. En: Grupo de trabajo de semFYC. Incapacidad Temporal: encrucijada ética, clínica y de gestión. Documento semFYC 12, 1999. p. 6.

8. Hermida L, Molina M, Martín J, Valero F. Evaluación de la Incapacidad Temporal por contingencias comunes en la Comunidad de Madrid. Periodo 1994-1999. Revista de Medicina y Seguridad en el Trabajo 2000; 186: 1-9.

9. Grupo de trabajo de incapacidad de la semFYC. ¿Incapaces de gestionar la incapacidad? Dimensión Humana 1998; 2 : 25-30.
10. Díaz R, Moya R, Vergara J, de Alarcón MC, Domínguez G. Análisis de la problemática derivada de los cambios legislativos en materia de Incapacidad Temporal. Semergen 1999; 24: 643-8.

11. Pineault R, Daveluy C. La Planificación Sanitaria: Conceptos, Métodos y Estrategias. Barcelona: Masson, SA, 1987. p. 297-8.

12. Altisent R, Huelin JV, Ariño M. Ética en la prescripción de la "baja laboral" por el médico de familia. Cuadernos de Gestión 1997; 3: 4-6.

13. INSALUD. Manual de Gestión de la Incapacidad Temporal. Madrid: Insalud, Servicio de Documentación y Publicaciones, 2001.

14. Sentencia de 15 de septiembre de $1998 \mathrm{n}^{\circ}$ 340/1998. Jurisdicción Civil. Audiencia Provincial de León. Sección Segunda.

15. Circular INSALUD 1/98, sobre gestión Lista de Espera Quirúrgica. Estudio del Programa de Lista de Espera Quirúrgica en el Área de Salud de Cuenca en 1999.

16. Blanco M, Candelas G, Jover JA, Hernández García C, Vargas E, Lázaro P. Economic evaluation of and intervention in patients with temporary work diseability of musculoskeletal origin. Arthritis \& Rheumatism 1999; 42 (Supl. 9): S387.

17. Gervás JJ, Del Burgo JL, Pérez Fernández $\mathbf{M}^{\mathrm{a}} \mathrm{M}$. La Historia Clínica. En: Gervás JJ. Los Sistemas de Registro en Atención Primaria de Salud. Madrid: Díaz de Santos, 1987. p. 25-90.

18. Circular INSALUD 1997, de 1 de enero, sobre control de la incapacidad temporal por enfermedad común y accidente no laboral. Presidencia Ejecutiva del INSALUD.

19. Borrell Carrió F, Molero García JM, Duque Valencia A, Amador Romero J, Tejedor Varillas A. Decálogo de propuestas para la mejora de la gestión de la incapacidad temporal. En: Grupo de trabajo de semFYC. Mejoras en la gestión de la incapacidad temporal. Documento semFYC 12, 1998. p. 9-11. 